introduction of more complex equipment. ${ }^{2}$ The need for speed in extractions became less pressing when apparatus could apparently be relied on to give precise mixtures to patients, but the newer techniques also had risks. As the late K. C. Macarthy frequently said, "No anaesthetic agent is safer than its administrator." In the 1930s the introduction of the short-acting barbiturates ${ }^{3}$ simplified the induction of the robust, resistant patient, and many short cases of extraction were completed entirely without the addition of any inhalation anaesthetic. The use of trichloroethylene as an adjuvant with nitrous oxide and oxygen became general in Great Britain during the second world war $^{5}$ and allowed smoother anaesthesia to ensue. Many anaesthetists became expert in its use, employing higher percentages of oxygen than were previously the rule in treating the ambulant dental case. But deaths continued to occur, even though they were rare in relation to the vast numbers of dental anaesthetics administered.

One school of thought ${ }^{6}$ blamed the sitting-up position usually adopted; another ${ }^{7}$ pointed to the unreliability of the "demand-flow" type of apparatus to deliver accurate mixtures of the two gases under clinical conditions. Certainly cerebral damage can occur during anaesthesia, especially in the sitting-up position. But it is doubtful if the vasovagal reflex that triggers off a fainting attack will act in an anaesthetized patient. However, many patients are in a state of acute apprehension on entering the dental surgery, especially if they have been kept waiting a long time in a crowded and badly ventilated waiting-room among loquacious fellowsufferers.

Such patients are pale and many have a hypotensive attack before the administration of the anaesthetic. From his traditional position the dental anaesthetist, standing behind the patient, may be unable to appreciate the onset of such a response, and if anaesthesia is started under such conditions the patient may be in grave peril. It is a wise anaesthetist who faces his patient in the dental chair and carries on a short conversation with him so that he can assess his reaction to the strain of the ordeal. If hypotension is suspected the induction should take place in the supine position after administration of oxygen. Or it may be advisable to postpone the operation to another occasion when the patient can be suitably sedated.

Recently J. B. Brierley and A. A. Miller ${ }^{\mathbf{3}}$ have described one case of fatal brain damage and admirably discussed the relevant literature. Undoubtedly the safest method of operating in the oral cavity is under endotracheal anaesthesia, which allows adequate packing and the use of the supine position. But the numbers of patients are too great for such a methodical approach.

The use of halothane as an adjuvant ${ }^{9}$ has made possible the maintenance of anaesthesia with high percentages of oxygen hitherto considered impracticable in mitrous-oxideoxygen mixtures. The use of premixed cylinders containing $50 \%$ of each gas in conjunction with halothane has been suggested. ${ }^{10}$ Demand-flow types of apparatus have been

1 Hewitt, F. W., Anaesthetics and their Administration, 1893. London.

2 McKesson, E., I., Brit. med. 7., 1926, 2, 1113.

- Weese, H., Dtsch. med. W schr., 1933, 59, 47.

- Lundy, J.'S., Proc. Mayo Clin., 1935, 10, 536.

- Hewer, C. L., Brit med. f., 1944. 1, 92.

- Bourne, J. G., Lancet, 1957, 2, 499.

Goldman, V., Brit. dent. J., 1958, 105, 160.

- Brierley, J. B., and Miller, A. A., Lancet, 1966, 2, 869.

- Goldman, V., Anesth. Analg. Curr. Res., 1959, 38, 192.

10 Latham, J., and Parbrook, G. D., Anaesthesia, 1966, 21, 472.

Lundy, J." S., Clinical Anesthesia, 1943, p. 512. Philadelphia. greatly improved, and two accurate machines are now readily available in Britain. The British Standards Institute is at present endeavouring to apply standards of performance to them-not only when supplied by the manufacturers but also when maintained by their service engineers. But despite such advances prolonged anaesthesia in the dental chair should not be attempted by the inexpert, nor should patients be submitted to it without a thorough medical examination. The use of intravenous anaesthesia for conservative dentistry, even when given so lightly that reflexes are not completely obtunded, must increase the risk to the patient of what would otherwise be a thoroughly safe procedure. The operatoranaesthetist is not, as a rule, the best person to judge a patient's medical fitness for prolonged administrations, and in the event of an emergency the patient's best interests could be served only by the presence of two qualified people.

Though neglected for many years as a branch of anaesthesia deserving special study, dental anaesthesia is now receiving its rightful attention, but much more clinical research is desirable. For instance, it is possible that intermittent intravenous anaesthesia may reduce the saturation of oxyhaemoglobin below $85 \%$-an undesirable consequence in patients who have any impairment of the cardiac output. If it in fact does so, then additional oxygen should be given to patients anaesthetized in this way, and here the premixed gases would prove valuable. Indeed J. S. Lundy ${ }^{11}$ recommended such supplementation with oxygen as far back as 1938. The drugs employed may have changed since then, but not the physiology of respiration nor the response of a patient to known respiratory depressants.

\section{Amines and Depression}

Depression in its many forms has been exhaustively studied for decades by psychiatrists of all kinds ; it has been a challenge to the drug firms ; and undeniably it has responded to treatment. Yet psychiatrists are still not agreed on the classification of its varied forms, its psychodynamics and aetiology, or its pathology. With his usual courage Professor Henry Miller recently entered the fray. ${ }^{1}$ The result was a lively, practical exposition of what may be called the contemporary materialist picture of endogenous depression. Professor Miller maintains that a neurologist may quite properly discourse on a psychiatric topic, such as endogenous depression. Certainly it is not so long since a large section of what is now psychiatric practice was in the hands of neurologists, and Professor Miller has already ventured, with telling effect, into the psychiatric field in his Milroy lectures on accident neurosis. ${ }^{2}$ And has he not got a diploma in psychological medicine?

Much of what Professor Miller presents on the treatment and symptomatology of depressive illness is accepted teaching, but not everyone would agree with his section on the nature of the disorder. He stresses the many causal factors-genetic, psychological, and temporal-which in differing combinations and valencies determine the clinical form, course, and responsiveness to treatment of attacks of depression in individuals, and he infers "a final common pathway of pathophysiological disturbance." The supporting evidence for this is chiefly biochemical, and as yet inconclusive. This is not surprising, since there is little justification at present for 
assuming that the popular clinical subdivisions into endogenous and other specified varieties correspond to biologically homogeneous groupings. Certainly it has not been possible to relate to a particular clinical form of the disease the slight changes observed in water and sodium metabolism of patients who are recovering under treatment. Nor can this be done for the high morning levels of cortisol in the plasma of depressed patients-a finding which corresponds to the increase in plasma-level of cortisol that occurs in normal persons subjected to environmental stress (such as an impending surgical operation, final examinations, rowing in a university boat race) or to experimental situations which induced anxiety and distress. ${ }^{3}$ Among depressed patients, ${ }^{3}$ those with retarded depression were found to have higher mean levels of cortisol than those who were agitated and weeping. Clinical improvement was associated with a fall in the plasma corticosteroid level, except in some chronically ill patients in whom with clinical improvement there was a rise in plasma corticosteroids. As usual in such studies the situation is complex and the physiological change seems not to be specifically related to the clinical symptoms.

The catecholamine hypothesis, to which Professor Miller referred, is stimulating but unconfirmed. When it was discovered that monoamine oxidase inhibitors could bring about recovery from a depressive illness, inquiry into the part played by cerebral monoamines in melancholia was quickened, and many studies have now suggested that these drugs act on a disorder of brain-amine metabolism. Depression, it is postulated, is associated with a deficiency of cerebral amines. Besides studies in man of the amount of noradrenaline and dopamine excreted in the urine during depression, direct measurement of catecholamines in the brain of animals has supplied some contingent evidence about their relation to emotion. Mice, rats, and guinea-pigs were subjected to stress by exposing them to fight with aggressive animals of the same species, giving them repeated electric shocks, immobilizing them, or stimulating the brain through implanted electrodes. ${ }^{4}$ In these animals a 10 to $30 \%$ decrement was found in the amount of noradrenaline in the brain. It was concluded that prolonged severe emotional upset activated a large number of cerebral neurones, which released noradrenaline; the concentration of serotonin and dopamine in the brain, however, did not alter. Unfortunately measurement of the amount of noradrenaline excreted in the urine has given discrepant results. This test is in any case potentially deceptive, since it is probable that only a small proportion of the noradrenaline liberated in the brain passes the bloodbrain barrier, the bulk of the noradrenaline in blood and urine deriving from the peripheral sympathetic nervous system.

The biochemical explanation designed to reconcile the antidepressant action of imipramine with the catecholamine hypothesis has been the occasion of further investigation of the metabolites of noradrenaline, particularly normetadrenaline and vanillylmandelic acid. J. J. Schildkraut and his colleagues at Bethesda have shown that as depressed patients improve while under treatment with imipramine there is a gradual increase in the amount of normetadrenaline excreted. They think it probable that this can be taken as an indication

1 Miller, H., Brit. med. 7., 1967, 1, 257.

Michael, R. P., and Gibbons, J. L., Int. Rev. Neurobiol., 1963, 5, 243.

- Bliss, E. L., and Zwanziger, J., f. psychiat. Res., 1966, 4, 189.

- Schildkraut, J. J., Green, R., Gordon, E. K., and Durell, J., Amer. f. Psychiat., 1966, 123, 690. of the amount of noradrenaline activity in the nervous system. ${ }^{\mathrm{s}}$

Incomplete and uncertain though our present information on these matters is, it warrants Professor Miller's judicious conclusion that the catecholamine hypothesis "furnishes a convenient frame of reference for both reflection and investigation in this important and absorbing field of medicine."

\section{Prognosis in Breast Cancer}

One way of estimating the prognosis of patients with breast cancer is to measure the corticosteroids excreted in the urine. ${ }^{1}$ In particular the urinary levels of aetiocholanolone (an androgen metabolite) and the 17-hydroxycorticosteroids have been measured and the measurements combined to make a "discriminant function" which was found to be helpful in forecasting response to treatment and prognosis.

The preoperative levels of these corticosteroids gave a reasonably accurate prediction of the response of a patient with advanced breast cancer to adrenalectomy or hypophysectomy. Further, when measured in patients who had undergone mastectomy for breast cancer the urinary corticosteroids gave a good indication of the chances of recurrence. ${ }^{2}$

Many of these findings have since been independently confirmed, ${ }^{3}$ but the procedures for measuring the steroids are so complex that they can be carried out only in specialized laboratories, and hence are of little direct clinical value. Recently H. Miller, J. A. Durant, A. G. Jacobs, and Jacqueline $\mathrm{F}$. Allison ${ }^{5}$ have reported an alternative method of using urinary corticosteroid measurements for predicting the response to the treatment of breast cancer. While employing the same concept of measuring androgens and corticoid metabolites, they have found that a discriminant based on a simple ratio of the pooled 11-deoxy-17-oxosteroids (these include aetiocholanolone but also other androgen metabolites) and the 17-hydroxycorticosteroids can be compared almost exactly with the original discriminant in which aetiocholanolone had to be measured specifically.

The original and the new discriminants were measured in 94 women with breast cancer and 55 healthy women. A remarkable correlation was noted both in the prediction of response of patients with advanced breast cancer to endocrine ablation and also in the variation of the discriminant value with age in normal women. The great advantage of this new approach is that the measurement of the total 11-deoxy-17oxosteroids is well within the compass of most hospital laboratories. The information, when available to those surgeons who perform adrenalectomy or hypophysectomy, can be used to predict the response of their patients.

It has also been hinted that the original discriminant might be a valuable tool to detect a group of women in the normal population who stand a high risk of developing breast cancer. ${ }^{\circ}$ This group could then be examined frequently, both clinically and by mammography. If a carcinoma developed it

\footnotetext{
Brit. med. F., 1965, 2, 1198.

Bulbrook, R. D., Hayward, J. L., and Thomas, B. S., Lancet, 1964, 1, 945 .

- Juret, P., Hayem, M., and Fleisler, A., 7. Chir. (Paris), 1964, 87, 409.

- Kumaoka, S., Abe, Ö., Sakauchi, N., Takatani, O., and Kusama, M. Abstr. Ninth Int. Cancer Congress, 1966, p. 653.

Miller, H., Durant, J. A., Jacobs, A. G., and Allison, J. F., Brit. med. f., 1967, 1, 147 .

Hayward, J. L., Proc. roy. Soc. Med., 1966, 59, 1204.

Philip, J. F., Brit. med. F., 1967, 1, 323.
} 